\title{
Eficacia de los opioides tópicos como analgésicos en enfermedades dolorosas cutáneas. Revisión de la literatura científica y propuesta metodológica para su evaluación clínica
}

\author{
G. Carvajal Valdy \\ Medicina Paliativa. Hospital William Allen. Turrialba, Costa Rica
}

Carvajal Valdy G. Eficacia de los opioides tópicos como analgésicos en enfermedades dolorosas cutáneas. Revisión de la literatura científica y propuesta metodológica para su evaluación clínica. Rev Soc Esp Dolor 2015; 22(1): 13-26.

\begin{abstract}
Introduction: Even though it is a common practice in some centers, there is still little evidence that justifies the use of topical opioid-based analgesics in skin and mucosal diseases.

Objectives: To review the available scientific literature that justifies the use of topical opioids in the treatment of painful skin and mucosal diseases. Evaluate the analgesic efficacy and adverse effects of topical opioids in the treatment of oral diseases and painful skin diseases.

Methods: An electronic search was performed using the following databases and search engines: EMBASE, Medline, SCIELO, LATINDEX, Current Controlled-Trials and academic Google to identify the literature on topical opioids in the treatment of painful skin and oral mucosa diseases. The selection method was based on the combination of different descriptors concerning topical opioids and pain using $\mathrm{MeSH}$ vocabulary. The search terms used were "opioid" "topic", "analgesic", "ulcer", "mucositis," "peripheral" and combinations of these words. Additional articles were retrieved from the reference lists of papers identified. The final search took place in July 2013. The inclusion criteria for the review were: Human studies, published in Spanish, French, English or German, containing main data on the efficacy of topical opioids as analgesics. We excluded from analysis studies that evaluated the effectiveness of opioids administered by invasive methods
\end{abstract}

Recibido: 04-08-13.

Aceptado: 20-11-13. (such intrapleural, intravesical, or intraarticular) or ophthalmic administration.

Results and conclusions: 28 publications were included from the search, 11 corresponded to prospective, randomized, controlled trials. The duration of randomized studies was short, with a following period of one week or less. There isn't however enough information to compare studies because of the heterogeneity of populations and treatments. The studies that controlled systemic levels of opioids comment conflicting results regarding the absorption of topical opioids through ulcerated skin, possibly associated with variables such as drug preparation, dosage, application extension and duration of treatment. Regarding adverse effects, these are minimal in appearance and limited to local reactions, and those studies that report these do not determine a definitive causal relationship.

Key words: Opioids. Topic. Analgesic. Ulcer. Mucositis.

\section{RESUMEN}

Introducción: aunque se trata de una práctica frecuente en algunos centros, es aún poca la evidencia que justifica el uso de formulaciones tópicas a base de opioides en el tratamiento analgésico de enfermedades dolorosas cutáneas y en mucosas.

Objetivos: realizar una revisión actualizada sobre la literatura científica disponible de la evidencia que justifica el uso de opioides tópicos en el tratamiento de enfermedades cutáneas y orales dolorosas. Evaluar la eficacia analgésica reportada y los efectos adversos de los opioides tópicos en el tratamiento de enfermedades cutáneas y orales dolorosas.

Métodos: se realizó una búsqueda electrónica utilizando las siguientes bases de datos y motores de búsqueda: EMBASE, Medline, SCIELO, LATINDEX, Current Controlled-Trials y Google académico, para identificar la literatura sobre opioides tópicos en el tratamiento de enfermedades dolorosas de la piel y la mucosa oral. El método propuesto para la selección de la muestra de trabajos está basado en la combinación de diferentes perfiles de búsqueda electrónica compuestos por descrip- 
tores referentes a opioides tópicos y tratamiento del dolor. Se construyeron diferentes sintaxis de búsqueda, considerándose ortodoxo utilizar términos obtenidos del vocabulario MeSH. Los términos de búsqueda utilizados fueron: "opioides", "tópico", "analgésico", "úlcera", "mucositis", "periférico" y combinaciones de estas palabras. Se identificaron los artículos adicionales a partir de las listas de referencias de los documentos recuperados, los artículos de revisión pertinentes y de revistas seleccionadas. La búsqueda final tuvo lugar en julio de 2013. Los criterios de inclusión para la revisión fueron: estudios en humanos, publicados en los idiomas español, francés, inglés o alemán, que contuvieran los datos principales sobre la eficacia de la aplicación tópica de opioides. Se excluyeron del análisis aquellos estudios que evaluaran la eficacia de opioides administrados por métodos invasivos (como vía intrapleural, intravesical, o intraarticular) o en administración oftálmica.

Resultados y conclusiones: se incluyeron 28 publicaciones a partir de la búsqueda, 11 correspondieron a estudios prospectivos, aleatorizados, controlados. La duración de los estudios aleatorizados fue corta, con un seguimiento inferior a una semana. No existe sin embargo suficiente información para comparar los estudios por la heterogeneidad de las poblaciones y de los tratamientos instaurados. Los estudios que controlaron niveles de opioides sistémicos comentan resultados contradictorios respecto a la absorción sistémica de los opioides a través de piel ulcerada, posiblemente asociado a variables como fármaco, preparación, dosis, extensión de la aplicación y duración del tratamiento. Respecto a los efectos adversos, estos son en apariencia mínimos y limitados a reacciones locales, y aquellos estudios que los reportan no determinan una relación de causalidad definitiva.

Palabras clave: Opioides. Tópico. Analgésico. Úlcera. Mucositis.

\section{ABREVIATURAS}

AINE: antiinflamatorios no esteroideos. MOR: Morphine opioid receptors. AMPc: adenosinmonofosfato cíclico. GTP: guanosin trifosfato. PI3K $\gamma$ : fosfatidilinositol 3-kinasa $\gamma$. PKG: protein kinasa G. AKT: serina treonina protein kinasa Akt. nNOS: óxido nítrico sintasa neuronal. NO: óxido nítrico. $\mathrm{K}_{\mathrm{ATP}}$ : canales de potasio sensibles a trifosfato de adenosina. SNC: sistema nervioso central. DMSO: dimetilsufóxido. HPLC: High-performance liquid chromatography. FAST: Functional assessment screening tool. EVA: escala visual analógica.

\section{INTRODUCCIÓN}

Aunque se trata de una especialidad en pleno desarrollo, la medicina del dolor sigue siendo un área difícil para la investigación (1). La heterogeneidad de la población atendida, dificultades en la evaluación y clasificación de los síntomas, reclutamiento insuficiente y alta tasas de abando- no que llevan a bajo poder estadístico son reconocidos problemas que contribuyen a las dificultades en la realización de proyectos de investigación robustos (2). No obstante, el impacto de las enfermedades dolorosas en los pacientes, cuidadores, personal y los servicios de salud hacen de esta un área que requiere más atención (3).

Un enfoque local en el tratamiento analgésico de enfermedades dolorosas consiste en aplicar medicamentos localmente en el sitio periférico de origen del dolor. Esto puede alcanzarse mediante la aplicación tópica de una crema, loción, gel, aerosol o parche para sitios somáticos o mediante la utilización de enjuagues en el caso de lesiones de la mucosa oral. Estos métodos de aplicación permiten una mayor concentración local de la droga en el sitio de iniciación del dolor y disminuyen los niveles sistémicos del fármaco a niveles mínimos o insignificantes, teóricamente reduciendo el riesgo de efectos adversos sistémicos. Otras posibles ventajas de las aplicaciones localizadas son la falta de interacciones farmacológicas, la falta de necesidad de titular la dosis hasta la tolerabilidad y -lo que no deja de ser relevante- la facilidad de uso, lo que facilita la adherencia. Es sin embargo predecible algún grado de absorción sistémica posterior a la administración local, especialmente con fármacos liposolubles y de bajo peso molecular; este grado de absorción sistémica debe siempre evaluarse durante el desarrollo de formulaciones tópicas, de igual manera, es importante una monitorización de los potenciales efectos adversos locales. En este punto es fundamental aclarar que, por definición, los medicamentos tópicos utilizados para controlar el dolor actúan localmente en tejidos blandos dañados o disfuncionales y que los sistemas tópicos de entrega difieren de los sistemas de entrega transdérmica en los que el tejido destino está inmediatamente adyacente al sitio de entrega, en lugar de utilizar la piel como un sistema alternativo de entrega sistémica (5-7). Es en este contexto que se han desarrollado formulaciones tópicas analgésicas, estas preparaciones -se cree- podrán evitar algunos problemas asociados con la vía oral, intravenosa, transdérmica o subcutánea, manteniendo una eficacia clínica satisfactoria.

\section{MECANISMOS DE ACCIÓN PERIFÉRICOS DE LOS OPIOIDES}

El sistema de opioides endógeno es uno de los principales sistemas que participan en mecanismos evolutivos fuertemente conservados como la percepción y modulación del dolor, y comportamientos de recompensa, adicción y miedo (7-9).

Los efectos centrales de los opioides en la transmisión de dolor por sus acciones en el asta dorsal de la médula espinal y tronco encefálico, entre otros sitios supraspinales, han sido reconocidos por más de tres décadas (10). Los receptores opiáceos y sus agonistas correspondientes 
son de suma importancia en la inhibición y la modulación del dolor, aunque no esenciales (11). Supraespinalmente, un número de regiones es sensible a la microinyección de morfina, incluyendo la sustancia gris periacueductal, el núcleo magno del rafe, el locus ceruleus y el núcleo reticular gigantocelular (12-15). A nivel espinal, agonistas opioides como la morfina actúan a través de receptores $\mu$ (MOR) localizados en el asta dorsal (16). A partir de esta base, durante mucho tiempo se ha postulado que la analgesia clínicamente suficiente se obtiene exclusivamente a través de la activación de los receptores opioides centrales. Se sabe ahora que los nociceptores cutáneos juegan un papel relevante en la nocicepción (17) y que receptores para opioides también están presentes en los terminales periféricos de fibras sensoriales cutáneas mielinizadas y pocomielinizadas (18). En 1979 Ferreira y Nakamura demostraron la obtención de analgesia periférica inducida por morfina en la hiperalgesia inducida por prostaglandina $\mathrm{E}_{2}$ en ratones (19); en 1987, el grupo de Russell en Alemania (18) demostró la presencia de receptores opioides en fibras nerviosas en la articulación de la rodilla del gato y evidenció que la administración de agonistas opioides -entre ellos la morfina- disminuía las descargas espontáneas de fibras sensoriales de diámetro pequeño. Este autor sugirió que los opioides pueden actuar sobre receptores periféricos, ejerciendo un efecto analgésico periférico. Desde la fecha, estudios en animales y humanos han demostrado la presencia de receptores opioides en fibras nerviosas cutáneas

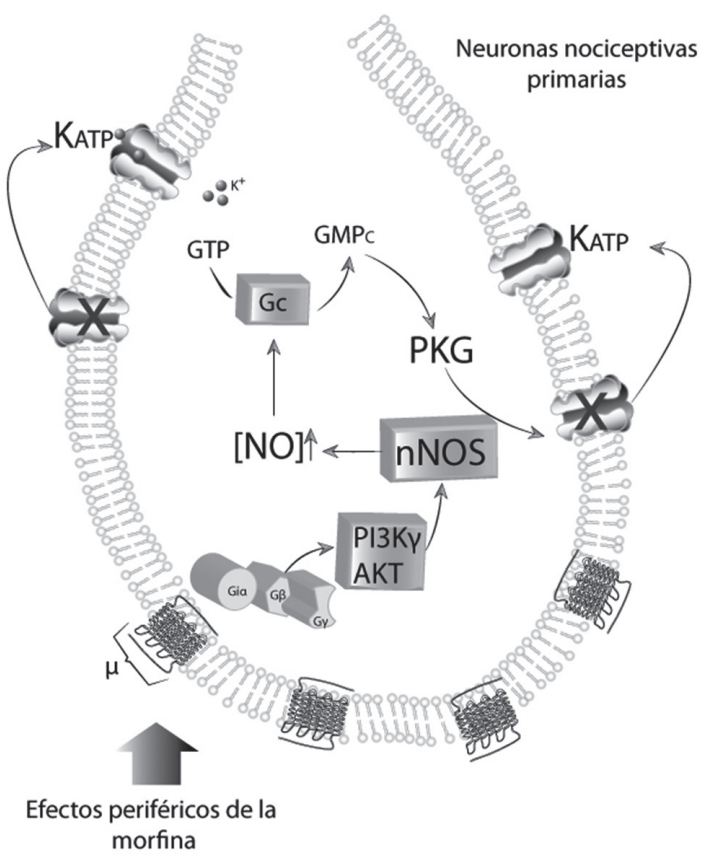

Fig. 1. Bases moleculares de la analgesia periférica inducida por morfina (25). de diámetro reducido (20), en hueso y tejido articular (21), queratinocitos (22) y células inmunológicas (23). Estos, y estudios similares sugieren un papel para los receptores periféricos, independiente de las acciones centrales (24).

\section{Mecanismos celulares}

Los efectos analgésicos periféricos de la morfina parecen depender de la activación de la vía de señalización $\mathrm{PI} 3 \mathrm{~K} \gamma / \mathrm{AKT} / \mathrm{nNOS} / \mathrm{NO} / \mathrm{K}_{\text {ATP }}(25)$, como se detalla en la figura 1 . Se ha demostrado en ratas que los canales de potasio GIRK2 son necesarios y suficientes para explicar los mecanismos periféricos de la analgesia inducida por opioides (26). Existe cada vez más evidencia experimental que sugiere que los receptores opioides periféricos se activan por cambios inflamatorios y que los opioides endógenos y exógenos pueden jugar un papel importante en la modulación del dolor y del proceso inflamatorio (24,27-30).

La activación de nociceptores en neuronas sensitivas primarias por opioides produce la activación de la vía PI3K $\gamma /$ AKT la cual podía estimular a la sintasa de óxido nítrico neuronal. El NO a través de la activación de cgmp/ PKG causaría la regulación en alzada de corrientes $\mathrm{K}_{\text {ATP }}$ facilitando la hiperpolarización celular.

\section{USO DE OPIOIDES TÓPICOS COMO ANALGÉSICOS}

En el contexto descrito en apartados previos se han desarrollado formulaciones tópicas basadas en opioides (31). Aunque su uso tradicional se remonta a larga data, como parte de la medicina ayurvédica (32), ha sido reciente la reintroducción del uso tópico de opioides a la formalidad de la medicina basada en evidencia, y el reporte de Back y Finlay publicado en 1995 constituye un primer esfuerzo reciente por notar las acciones terapéuticas del uso de opioides tópicos en enfermedades dolorosas cutáneas (33). Los principales opioides estudiados en este sentido son morfina, metadona, diamorfina y buprenorfina, pero existen reportes anecdóticos de uso de oxicodona y meperidina.

Es importante destacar que no existe actualmente una formulación tópica a base de opioides comercialmente disponible, sino que estas son prescritas y administradas a partir de formulaciones magistrales particulares para cada centro médico. Así, en el Centro Nacional de Control del Dolor y Cuidados Paliativos de Costa Rica se prescribe un gel de morfina al 0,2 \% con base hidrosoluble de propilenglicol, pero es posible encontrar reportes internacionales muy heterogéneos sobre el uso de estas presentaciones, sobre lo cual se escribirá más adelante.

Respecto a la estabilidad de las formulaciones utilizadas, se han estudiado distintas combinaciones de opioides 
Rev. Soc. Esp. del Dolor, Vol. 22, N. ${ }^{\circ}$ 1, Enero-Febrero 2015

y vehículos; una de ellas es el hidrogel Intrasite ${ }^{\circledR}$ (Hidrogel amorfo; Smith\&Nephew, Inglaterra), un hidrogel que contiene agua, propilenglicol y carboximetilcelulosa, cuya aplicación principal se da en úlceras por decúbito. Este hidrogel ha demostrado mantener la estabilidad de soluciones de morfina y buprenorfina con concentraciones de $1,25 \mathrm{mg} / \mathrm{ml}$, durante al menos 28 días (34). Otra base utilizada en el caso del Western General Hospital en Edimburgo es el Instillagel ${ }^{\circledast}$ (gel con lidocaína al $2 \%$, clorexidina $0,25 \%$ como ingredientes activos, FARCOPHARMA). Abdelmageed demostró que el gel de morfina con base de Instillagel ${ }^{\oplus}$ mantenía su estabilidad $(98,1 \%$ a 103,2 \% de la concentración inicial) a 37 grados Celsius por hasta 7 meses (35). Para otras bases que han sido utilizadas como Stomahesive ${ }^{\circledR}$ (Convatec, EE. UU.) y DuoDerm $^{\circledast}$ (Convatec, EE. UU.), las cuales se componen de carboximetilcelulosa sódica, pectina y gelatina en una base alcohólica, Lavasept ${ }^{\circledR}$ (Fresenius AG, Alemania) compuesto por polihexamida, o el organogel plurónico de lecitina (gel PLO), no existe ningún estudio de estabilidad farmacológica publicado. Un estudio reciente sugiere que la utilización de polihexamida en lugar de geles a base de polietilenglicol podría disminuir la aparición de sensibilización alérgica (36). En particular llama la atención que se utilice el gel PLO con intención de tratamiento tópico, ya que este fue diseñado para mejorar la absorción de medicamentos a través de la epidermis, lo cual contraindicaría su acción tópica, tratándose de una tentativa de administración transdérmica (37).

\section{ESTUDIOS EN MODELOS ANIMALES}

La mayoría de los estudios preclínicos que evalúan el uso de opioides tópicos se basan en el modelo clásico de la prueba del coletazo, en inglés conocido como "tail flick test" $(38,39)$. En su estudio en ratones, utilizando el modelo de la prueba del coletazo, Kolesnikov y cols. (40) demostraron que la inyección local de morfina subcutánea en la cola de la rata producía una analgesia dosis-dependiente, con una dosis efectiva de 4,5 pg, y que las dosis de morfina activas localmente eran inactivas de manera sistémica. De igual manera, la analgesia obtenida desaparecía al mover la fuente de calor radiante a $1 \mathrm{~cm}$ del sitio de inyección local. Esta analgesia era reversible por la utilización de naloxona, un inhibidor competitivo de receptores MOR

En un estudio posterior, Kolesnikov y cols. (41) confirmaron nuevamente que la inmersión de la cola de un ratón en una solución de dimetilsufóxido (DMSO) con morfina producía una analgesia dosis dependiente reversible por naloxona limitada a la porción de la cola expuesta al opioide no explicable por el medio difusor (DMSO). El estudio reportó resultados similares con otros dos agonistas opioides: levorfanol y buprenorfina.

\section{EVIDENCIA CLÍNICA EN HUMANOS}

A pesar del extensivo uso de los opioides en la medicina, los ensayos clínicos que evalúan esta vía de administración son limitados tanto en número como en calidad metodológica, correspondiendo la mayoría de las publicaciones a reportes aislados de casos o a series de casos de escala reducida, con diseños metodológicos abiertos, frecuentemente sin grupos control adecuados, como se demostrará posteriormente. De igual manera, existe poca información científica disponible sobre la farmacocinética de estos medicamentos al ser utilizados tópicamente.

\section{ESTUDIOS FARMACOCINÉTICOS}

En humanos, en un estudio prospectivo, dirigido por Ribeiro (42) se investigó la biodisponibilidad de morfina tópicamente aplicada a úlceras cutáneas; en este estudio se incluyeron seis pacientes adultos de un hospicio. Los pacientes fueron elegibles si tenían úlceras de piel (mayores de $2 \mathrm{~cm}$ de diámetro y $0,5 \mathrm{~cm}$ de profundidad) que no estaban infectadas o cubiertas por tejido necrótico. Los pacientes no podían recibir concomitantemente morfina, codeína, diamorfina o hidromorfona, pero el fentanilo y el tramadol fueron permitidos, ya que no interferían con el ensayo HPLC para la morfina. Estos recibieron $10 \mathrm{mg}$ de sulfato de morfina tópicamente en gel Intrasite ${ }^{\circledR}$ y morfina sulfato $10 \mathrm{mg}$ por vía subcutánea durante 4 horas, separados por al menos 48 horas, en orden aleatorio. Se cuantificaron los niveles plasmáticos de morfina, morfina6-glucurónido, y morfina-3-glucurónido, utilizando HPLC. En cinco pacientes los niveles fueron indetectables. En un paciente (con la úlcera de mayores dimensiones), se detectó morfina y uno de sus metabolitos: morfina-6-glucurónido. Este paciente tenía una úlcera de dimensiones considerables $60 \mathrm{~cm}^{2}$ en comparación con un promedio de $12,8 \mathrm{~cm}^{2}$ en los otros 5 pacientes estudiados. La biodisponibilidad de la morfina y de la morfina-6-glucurónido en este paciente fue de $20 \%$ y $21 \%$, respectivamente. Esto, a criterio de los investigadores, sugiere que cuando se aplica tópicamente a úlceras cutáneas de tamaño reducido, la morfina no se absorbe en cantidades significativas, sugiriendo que cualquier efecto analgésico podría estar mediado localmente. Una limitación importante de este estudio -cuyo diseño tenía objetivos farmacocinéticos únicamente- es que no se comparó el nivel de analgesia obtenido entre ambas vías de administración (opioide sistémico vs. opioide tópico), aunque el autor reporta que la dosis tópica utilizada fue de $10 \mathrm{mg}$, que es la que se utiliza comúnmente. Esto limita la posibilidad de concluir en términos de eficacia analgésica de la formulación utilizada. Por otro lado, a pesar de tratarse de pacientes debilitados, no se reportó la función renal estimada, la cual podría alterar la cinética del opioi- 
de evaluado, aunque según los datos reportados la vida media de eliminación de la morfina se encontraba dentro de lo reportado por otros estudios $(2,5 \pm 0,3$ horas $)$, lo cual sugiere que no había un deterioro importante en la tasa de filtración glomerular.

Watterson y cols. (42) en su estudio en 2 niñas con epidermólisis bullosa reportan que sí se detectaron niveles sistémicos de morfina, después del tratamiento tópico prolongado (meses) con morfina al $0,1 \%$ en gel Intrasite ${ }^{\circledR}$ diario (en lesiones de extensión no reportada), pero que estos eran inferiores a los niveles reportados para analgesia sistémica (no se detalla esta información).

En otro estudio en voluntarios sanos, Paice y cols. (44) evaluaron la biodisponibilidad de la morfina tópica aplicada en organogel plurónico de lecitina (gel PLO) en la piel intacta de voluntarios. Se realizó un estudio aleatorizado, controlado con placebo, doble ciego, cruzado de cinco voluntarios, siendo asignados los voluntarios al azar para recibir ya sea $1 \mathrm{ml}$ de morfina en concentración $10 \mathrm{mg} /$ $\mathrm{ml}(1 \%)$ en una base de organogel plurónico de lecitina aplicado en la muñeca y $1 \mathrm{ml}$ de solución salina normal administrado por vía subcutánea, o $1 \mathrm{ml}$ de placebo y $1 \mathrm{ml}$ de morfina subcutánea, $3 \mathrm{mg} / \mathrm{ml}$, invirtiendo la secuencia posteriormente. No se logró detectar morfina en las muestras de plasma de pacientes posterior a la administración tópica de morfina en 2 de los pacientes, y en 3 de ellos se detectaron niveles inferiores al nivel de cuantificación posible para la prueba $(0,5 \mathrm{ng} / \mathrm{ml})$, niveles muy inferiores a las concentraciones que se han descrito para analgesia sistémica $(10 \mathrm{ng} / \mathrm{ml})$. Esto a pesar que la concentración utilizada $(1 \%)$ es superior a la clínicamente reportada $(0,1 \%-0,5 \%)$. Estos resultados demuestran que la formulación tópica de morfina en concentración del $1 \%$ no se absorbe eficientemente a través de piel intacta.

En el 2005, Gallagher (45) demostró una absorción sistémica estimada de 0,3 a $4 \%$ de la dosis administrada, para dosis diarias de metadona de $25 \mathrm{mg}$, aplicada a úlceras por decúbito. Posteriormente, en el único estudio publicado sobre farmacocinética de la metadona tópica, Sylvester y cols. (46) compararon la absorción oral de la metadona con la absorción tópica (10-45 mg/día como polvo disuelto en etoxidiglicol en gel PLO) a través de piel intacta en pacientes con deterioro cognitivo severo, tratados por 7 días. Ellos observaron que 18 de 20 mediciones en 9 pacientes que recibieron metadona tópica fueron idénticas a las observadas en el grupo control. Únicamente un paciente que recibió metadona tópica tuvo concentraciones medibles y este tuvo una concentración promedio en las mediciones de 25,8 ng/ml (18-35 ng/ml). Este estudio sin embargo tiene grandes limitaciones: por un lado la población seleccionada limita la posibilidad de una evaluación más objetiva del dolor percibido por los pacientes (en promedio una edad de 90 años, y la totalidad de los pacientes tenían demencia de alguna etiología, con estadios FAST avanzados, siendo la titulación de la analgesia dependiente de comportamiento como quejidos, facies de dolor o rigidez). Por otro lado, la ausencia de un grupo control válido (un único voluntario sano), y el hecho de que la metadona tópica fue aplicada a un sitio de piel intacta, impiden concluir que existan diferencias respecto a un placebo. Como conclusión válida de orden farmacocinético, el estudio demuestra que la absorción transdérmica de dosis de metadona inferiores a $45 \mathrm{mg}$ es nula.

Los trabajos comentados previamente en general reflejan que la absorción de los opioides estudiados para aplicación tópica -metadona y morfina- es baja y que la obtención de niveles asociados con analgesia sistémica es improbable si se utilizan sobre piel íntegra o sobre lesiones no extensas. De aplicarse en lesiones cutáneas, su absorción depende de la extensión de las lesiones, siendo insignificante para la morfina al 0,1\% en gel Intrasite ${ }^{\circledR}$ en lesiones de tamaño reducido $\left(<13 \mathrm{~cm}^{2}\right)$ la cual, como se verá más adelante, es la formulación más frecuentemente utilizada y estudiada. Aunque no es posible realizar recomendaciones generales basadas en los casos específicos que han sido descritos hasta la actualidad.

\section{ESTUDIOS DE EFICACIA CLÍNICA}

La mayoría de la evidencia disponible en la literatura científica corresponde a reportes de casos aislados o a series de tamaño reducido (menos de diez casos reportados) lo cual dificulta la extracción de conclusiones aplicables a grupos poblacionales amplios. Aunque la totalidad de los casos reportados supera ya 400 pacientes con diferentes tipos de heridas superficiales agudas y crónicas, que sugieren que la aplicación tópica de un opiáceo en un gel puede producir una reducción en el nivel de dolor percibido en algunas enfermedades dolorosas, debe sin embargo asumirse la presencia del sesgo de publicación (47).

Los reportes de casos siguen constituyendo la base de la evidencia disponible, y aunque débil, con frecuencia representa el primer paso de toda investigación. Llama la atención la heterogeneidad de formulaciones estudiadas, en términos de población reportada (pacientes ambulatorios, pacientes internados), de la etiología de las causas del dolor (úlceras de etiología vascular, neuropática, reumática, por decúbito, tumorales), de los fármacos utilizados (morfina, metadona, diamorfina, oxicodona, meperidina), de las bases utilizadas como solventes (agua destilada, Instillagel $^{\circledR}$, Intrasite ${ }^{\circledR}$, Somahesive $^{\circledR}$, DuoDerm $^{\circledR}$, Lavasept ${ }^{\circledR}$, hidrocoloides, soluciones con diversos fármacos como antibióticos), aunque respecto a estas únicamente se ha demostrado la estabilidad de la morfina en gel Intrasite ${ }^{\circledR} \mathrm{y}$ de la morfina y la diamorfina en Instillagel ${ }^{\circledR}$.

Algunos estudios incluidos en este trabajo, como se detalla más adelante, reportan variables como inicio de la 
analgesia o persistencia de esta, sin embargo lamentablemente el grueso de los reportes carecen de análisis estadístico y de escalas objetivas de reporte del dolor como la escala visual analógica (EVA), haciendo imposible la comparación intrapaciente e interpaciente. Otra variable que podría dificultar la comparación de los estudios es el uso concomitante de opioides sistémicos, que constituye un fenómeno frecuente en los reportes evaluados, ya que los opioides tópicos se "agregan" al tratamiento de base. No se detalla en nuestro trabajo sobre los reportes de casos o las series debido a la heterogeneidad de estos en términos de la amplitud de las variables evaluadas y la poca sistematización que en general se observa, sin embargo la tendencia clara es hacia el alivio por periodos prolongados, aunque es difícil evaluar el sesgo de publicación (48).

En general, podrían clasificarse los estudios en 3 grandes grupos de patología: dolor por lesiones cutáneas crónicas, dolor por lesiones cutáneas agudas, y dolor por mucositis inducida por quimio y radioterapia.

\section{Dolor por lesiones cutáneas agudas}

Este subgrupo específico ha sido abordado en los estudios de Long (49), Welling (50) y Skiveren (51). En el 2001 Long inició un estudio con 4 pacientes aleatorizados a recibir tratamiento analgésico sistémico convencional y sulfadiazina argéntica $v s$. gel de sulfadiazina con morfina al $0,1 \%$, por hasta tres días, sin embargo por su bajo reclutamiento no le fue posible obtener resultados estadísticamente significativos, aunque hubo una tendencia en los pacientes que recibieron gel de sulfadiazina y morfina hacia la obtención de analgesia superior y el menor consumo de opioides sistémicos. Welling, estudiando los efectos de la morfina tópica al 0,07 \% en quemaduras de espesor parcial superficial en 49 pacientes aleatorizados en un grupo de apósito Jelonet ${ }^{\circledR}$, un grupo de gel placebo y un grupo de gel de morfina, no observó diferencias estadísticamente significativas entre los puntajes de dolor o calificaciones de comodidad de los tres tratamientos, aunque el subgrupo de morfina reportó un consumo de paracetamol o ibuprofeno menor a los otros dos. Finalmente, el estudio de Skiveren ha sido incluido en esta categoría aunque no aborda específicamente el uso tópico de morfina en tratamiento analgésico de quemaduras, utiliza un modelo que guarda similitudes: la terapia fotodinámica. En su estudio, en el cual se incluyeron 28 pacientes previos a la administración de terapia fotodinámica por lesiones malignas o premalignas de piel, el autor concluye que no existe diferencia estadística entre el grupo de tratamiento con morfina al $0,3 \%$ y el grupo placebo. En este estudio sin embargo llama la atención que la administración del gel haya sido 15 minutos previos a la iluminación, ya que se ha comentado en apartados anteriores la necesidad de presencia de inflamación para que exista eficacia de la analgesia periférica por opioides.

\section{Dolor por lesiones cutáneas crónicas}

Los reportes y las series de casos sobre esta categoría específica de lesiones son abundantes: desde el primer reporte de 5 pacientes tratados con morfina tópica por parte de Back y Finlay en 1995 (33) ha habido 92 casos en total, con limitaciones metodológicas propias de este tipo de reporte para evaluar la eficacia del alivio y la duración de este. Una única revisión sistemática ha sido realizada hasta la fecha (52), todos los estudios incluidos en esta fueron analizados en nuestro trabajo, a los cuales se les adicionó reportes no incluidos por los criterios de selección de Zeppetella y cols., y otros que fueron publicados posteriormente al periodo de corte (agosto de 2006). En nuestro trabajo, de las 28 publicaciones incluidas, 11 eran estudios aleatorizados y controlados, y de estos 5 específicamente evalúan la aplicación de un opioide en lesiones cutáneas crónicas como úlceras vasculares, tumorales o por decúbito. Tomando en cuenta la evidencia científica disponible no es posible realizar un metaanálisis a causa de la heterogeneidad de los estudios. Es sin embargo posible reconocer que existe una clara tendencia de los reportes a promover la morfina o la diamorfina tópica como una opción terapéutica válida y eficaz en pacientes con úlceras crónicas -este subgrupo específico de pacientes que constituye la mayoría de la población en la que se prescriben estos fármacos-. En esta categoría de lesiones cutáneas crónicas, la evidencia disponible se conforma fundamentalmente de series y reportes de casos, y la serie de casos con el mayor número de pacientes es la de Huptas y cols. (36), en la que en pacientes con úlceras cutáneas de diversa etiología (la mayoría con úlceras venosas de miembros inferiores) demostraron al utilizar morfina al $0,125 \%$ en gel neutro a base de agua, una disminución significativa $(\mathrm{p}=0,0001)$ en el dolor asociado a esta condición (EVA basal, EVA a las 24 horas post tratamiento 4,5 $\pm 2,4$, siendo la reducción absoluta de dolor entre 1-8 y un promedio de $3,8 \pm 1,7)$. Este estudio carece de grupo control, lo que dificulta que estos resultados positivos sean generalizados a la práctica clínica.

Entre los estudios de mayor calidad metodológica destacan los trabajos realizados en pacientes con úlceras por presión por Flock (53), Zeppetella (54) y Vernassiére (55), los dos primeros con resultados positivos utilizando morfina y diamorfina respectivamente al $0,1 \%$ en una aplicación diaria en gel Intrasite ${ }^{\circledR}$. El tercero sin embargo no pudo confirmar estos resultados, demostrando que la analgesia obtenida con morfina al $0,125 \%$ en gel Intrasite ${ }^{\circledR}$ no superaba al control con placebo. En pacientes con úlceras arteriales, los dos estudios disponibles han sido realizados por Jansen $(56,57)$ y no 
se obtuvo un efecto analgésico relevante después de la administración de morfina tópica al $0,1 \%$ o $0,5 \%$ en gel Intrasite ${ }^{\circledR}$ en pacientes con úlceras arteriales dolorosas. El autor de ambos trabajos sugiere que esto podría deberse a la ausencia de inflamación local o la naturaleza mixta (nociceptivo-neuropático) del dolor percibido. Un aspecto fundamental que no ha sido abordado por estos estudios es la tolerancia a largo plazo y sus efectos sobre la curación de las heridas tratadas. Sin duda esto deberá ser evaluado en estudios posteriores.

\section{Mucositis inducida por quimioterapia y radioterapia}

En el subgrupo específico de tratamiento de la mucositis de diversa etiología, destacan los estudios aleatorizados y controlados de Cerchietti $(58,59)$ y de Vayne $(60)$. En los dos primeros, Cerchietti demostró inicialmente la superioridad de un enjuague de morfina (sulfato) $0,2 \%$ en enjuague bucal de $15 \mathrm{ml}$ cada 3 horas, 6 veces al día sobre el enjuague bucal estándar (difenhidramina, lidocaína e hidróxido de aluminio) en un grupo de 26 pacientes; posteriormente demostró en un grupo de 10 pacientes la superioridad analgésica de una solución de morfina al 0,2 \% sobre una al $0,1 \%$, sin que ello se tradujera en absorción sistémica del medicamento; y posteriormente evidenció la reducción significativa y persistente de la analgesia obtenida con una solución de morfina al 0,2 \% en 22 pacientes con mucositis inducida por quimio/radioterapia (grado II). Cabe destacar que el investigador comenta un caso aislado de mucositis por herpes simplex con pobre respuesta al enjuague con morfina al 0,2\%, lo cual podría explicarse por el importante componente neuropático de esta condición. Esto sin embargo deberá ser demostrado. Por otro lado el estudio de Vayne y cols. no pudieron obtener los mismos hallazgos, con significancia estadística, por una muestra de pacientes reducida. En el 2010 Saroja y cols. (61) reportaron 10 casos de pacientes tratados con enjuagues de morfina al $0,3 \%$ en enjuagues de $15 \mathrm{ml}$ cada $2 \mathrm{~h}$ por $48 \mathrm{~h}$, en todos los casos los pacientes obtuvieron analgesia satisfactoria $60 \mathrm{minu}-$ tos post-tratamiento (aunque no se objetivó con una escala estandarizada).

La heterogeneidad de los diseños de estudio dificulta la comparación de estos. Los reportes (ensayos clínicos, estudio de caso, serie de casos), varían en duración, uso de analgésicos (opioides sistémicos, dosis), medio de base, intervalo de administración, características de la herida y las variables estudiadas diferentes (alivio del dolor, disminución del consumo de analgesia sistémica, duración de la analgesia). Queda sin embargo claro que en enfermedades como úlceras por decúbito y mucositis inducida por quimio/radioterapia la tendencia es hacia la producción de analgesia satisfactoria por periodos prolongados. En la tabla I se resumen los estudios incluidos en nuestra investigación.

\section{CONCLUSIONES}

La evidencia científica disponible sobre la eficacia clínica del uso de opioides tópicos es escasa y heterogénea, correspondiendo el grueso de esta a reportes de casos y series de tamaño reducido, con ensayos clínicos en poblaciones heterogéneas y de diseño variado que dificulta su comparación. Queda sin embargo claro que en enfermedades como úlceras por decúbito y mucositis inducida por radio/quimioterapia, la tendencia es hacia la producción de analgesia satisfactoria por periodos prolongados que superan la vida media de los fármacos utilizados.

En término de efectos adversos, la aplicación tópica rara vez produce reacciones no deseadas y la mayoría de los reportes de estas no tienen una asociación definitiva. Aunque los estudios en animales sugieren que los opioides tópicos pueden retrasar el proceso normal de la cicatrización de heridas asépticas, la relevancia de esto en la práctica clínica en los seres humanos es desconocida y no existe evidencia clínica sobre su efecto en heridas crónicas en seres humanos. Tomando en cuenta esto, será necesario seguir estudiando esta hipótesis para caracterizar completamente los efectos de la morfina tópica en heridas con inflamación crónica y evaluar la posibilidad de efectos deletéreos sobre la cicatrización, la cual no ha sido confirmada.

En resumen, esta revisión concluye que hay documentación científica que apoya el uso de morfina y diamorfina $0,1 \%$ en gel Intrasite ${ }^{\circledR}$ en aplicación diaria tópica en enfermedades cutáneas dolorosas crónicas exceptuando las úlceras arteriales, al igual que el uso de enjuagues de morfina al 0,1 y $0,2 \%$ en aplicaciones de $15 \mathrm{ml}$ cada 3 horas para mucositis inducida por quimio/radioterapia; estas recomendaciones se basan sin embargo en ensayos clínicos aislados con población reducida.

Deberán realizarse ensayos clínicos aleatorizados y controlados con poder estadístico suficiente que evalúen la acción de un opioide específico en una base que haya demostrado compatibilidad y estabilidad farmacológica, en un tipo específico de lesiones cutáneas (úlceras vasculares, tumorales, reumatológicas, por presión o mucositis), que documenten variables como obtención y duración de la analgesia, consumo de analgesia sistémica, efectos adversos locales, tolerancia a largo plazo, satisfacción del paciente, fármaco de elección, dosis y frecuencia de administración. Estos estudios deberán demostrar la ausencia de absorción sistémica relevante. 


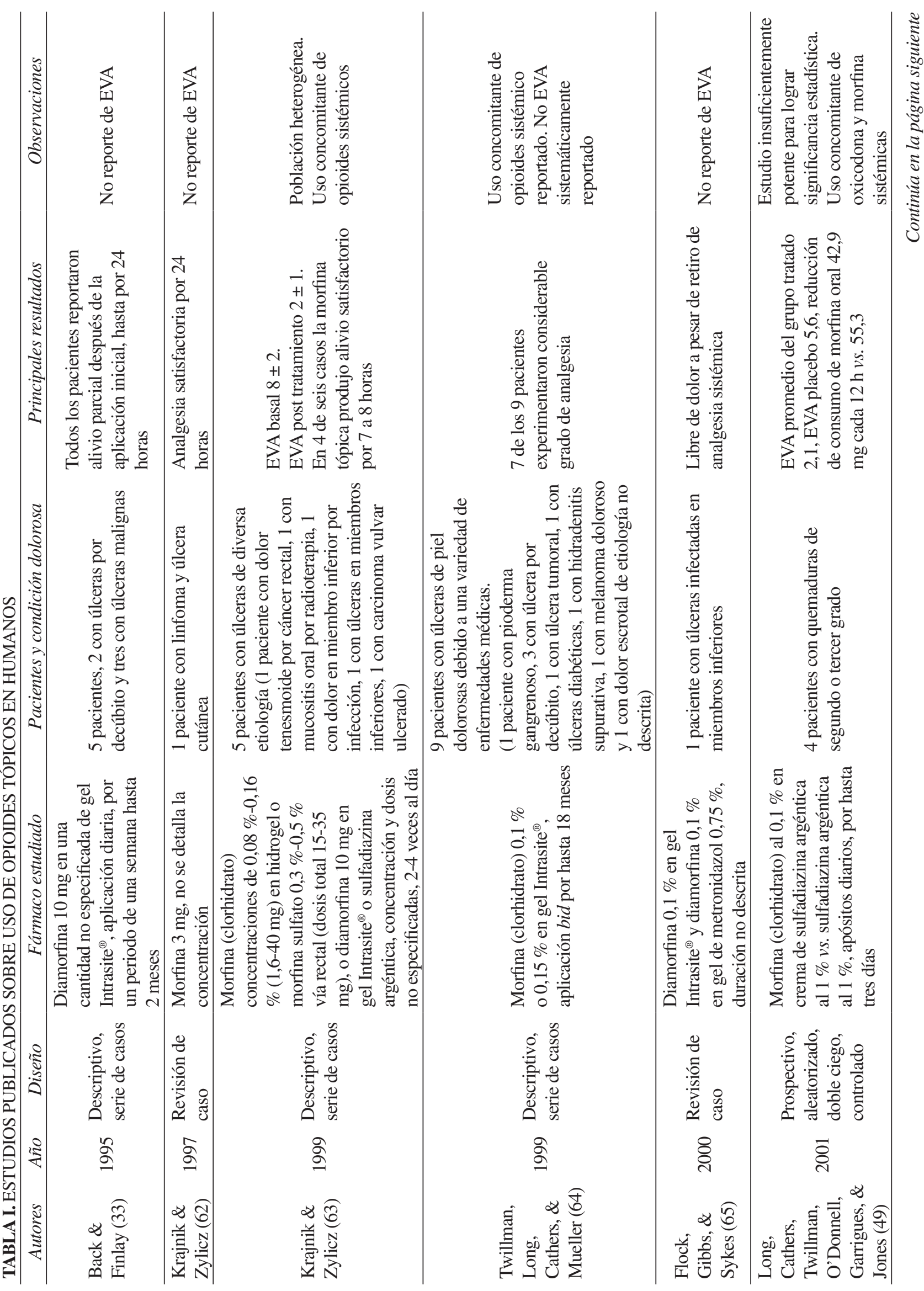


EFICACIA DE LOS OPIOIDES TÓPICOS COMO ANALGÉSICOS EN ENFERMEDADES DOLOROSAS CUTÁNEAS.

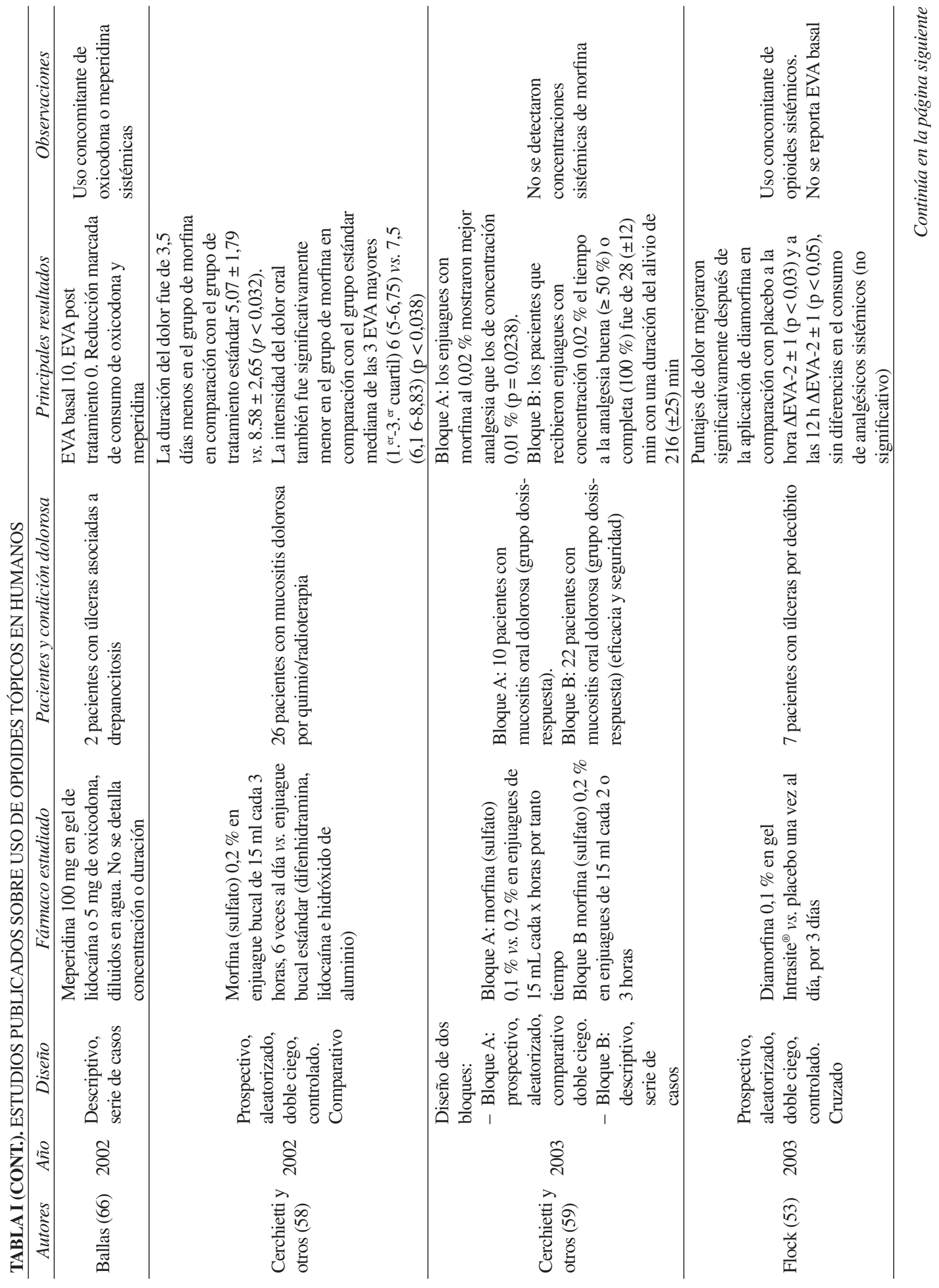




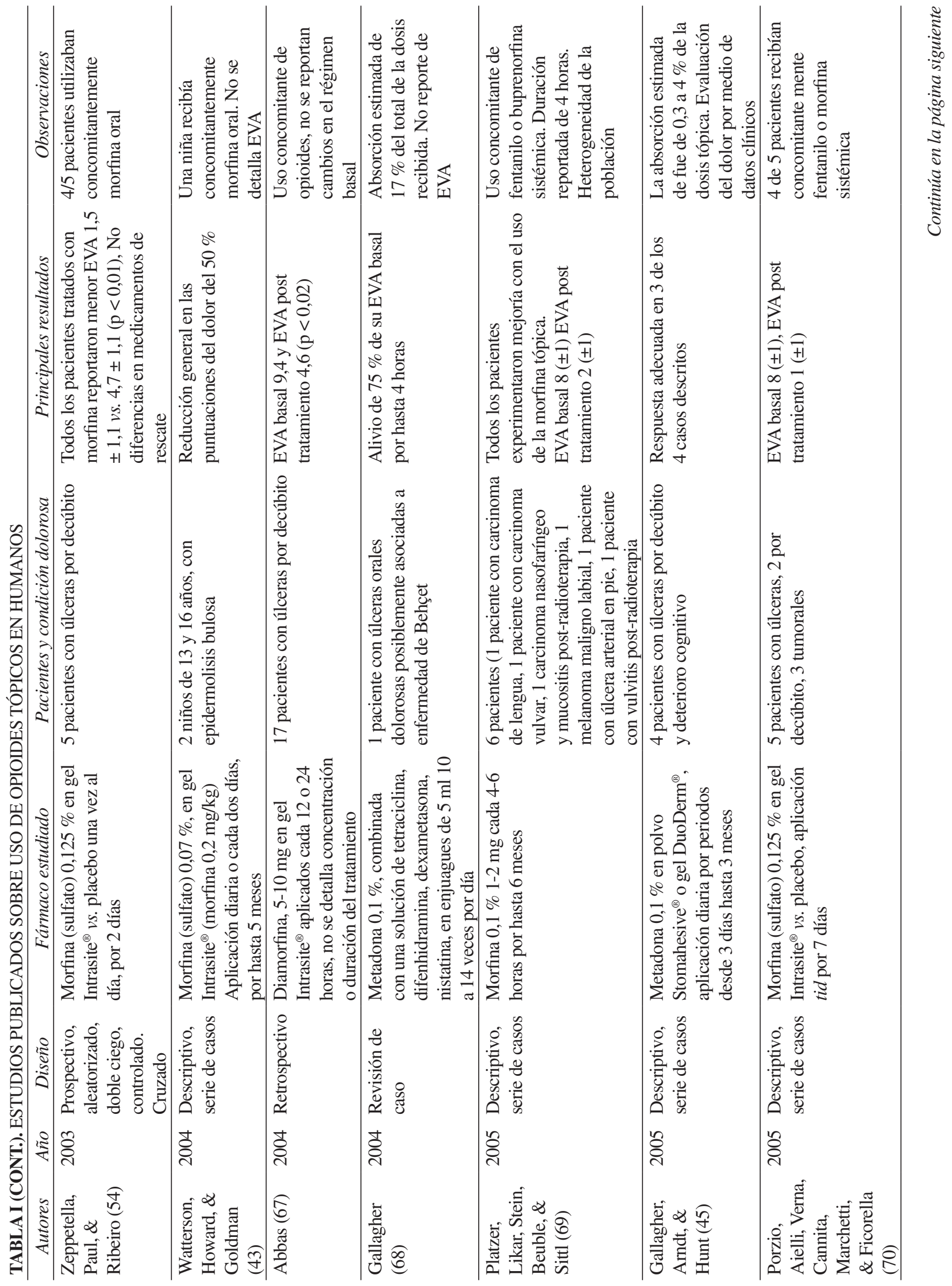


EFICACIA DE LOS OPIOIDES TÓPICOS COMO ANALGÉSICOS EN ENFERMEDADES DOLOROSAS CUTÁNEAS.

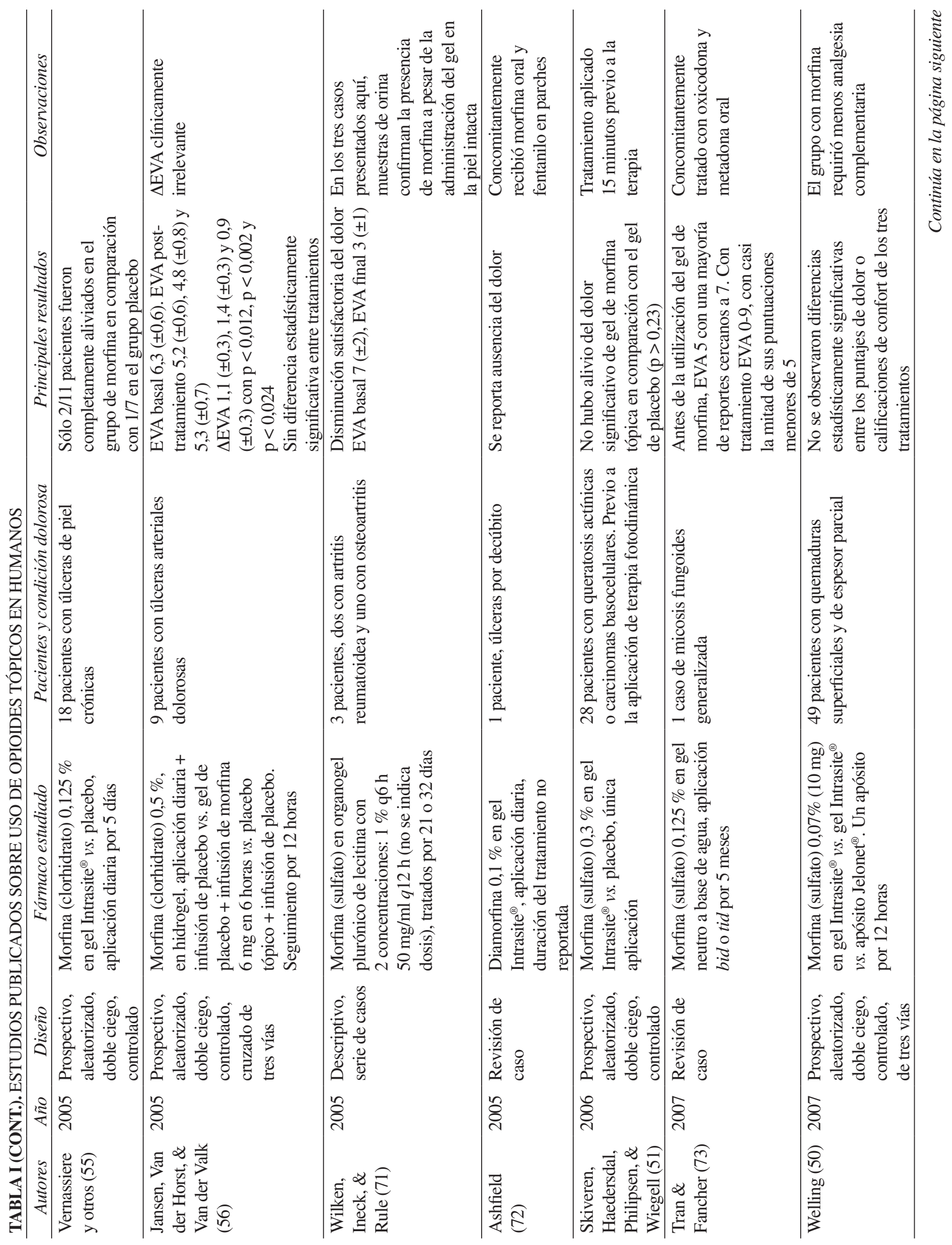




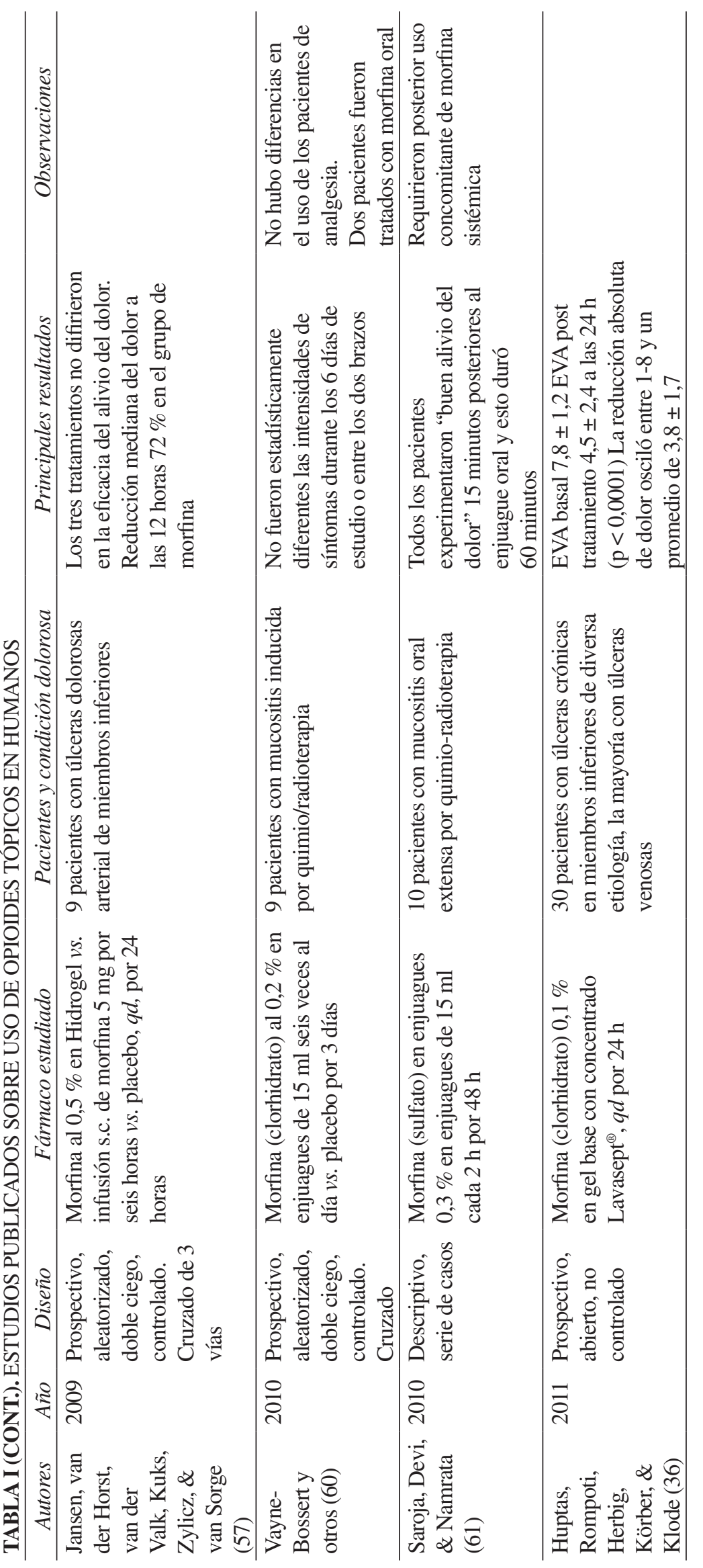




\section{CORRESPONDENCIA:}

Gabriel Carvajal Valdy

Medicina Paliativa

Hospital William Allen

Turrialba, Costa Rica

e-mail: gabrielcarvajalv@yahoo.com

\section{BIBLIOGRAFÍA}

1. O'Connor A, Dworkin R. Clinical trial design for chronic pain treatments. Evidence-based chronic pain management. Oxford: Blackwell Publishing Ltd; 2010.

2. Ballantyne JC. Pain medicine: Repairing a fractured dream. Anesthesiology 2011;114(2):243-6.

3. The Lancet. Managing pain e ectively. The Lancet 2011:2151.

4. McCleane G. Topical application of analgesics: A clinical option in day case anaesthesia? Curr Opin Anaesthesiol 2010;23(6):704-7.

5. Rao P, Mohamed T. Current and emerging "at-site" pain medications: A review. J Pain Res 2011;4:279-86.

6. Stanos S. Topical agents for the management of musculoskeletal pain. J Pain Symptom Manage 2007;33(3):342-55.

7. Sawynok J. Topical and peripherally acting analgesics. Pharmacol Rev 2003;55(1):1-20.

8. Petrovic P, Pleger B, Seymour B, Klöppel S, De Martino B. Blocking central opiate function modulates hedonic impact and anterior cingulate response to rewards and losses. $\mathbf{J}$ Neurosci 2008;28(42):10509-16.

9. Lehner M, Wislowska-Stanek A, Maciejak P, Szyndler J, Sobolewska A, Krza cik P, Pła nik A. The relationship between pain sensitivity and conditioned fear response in rats. Acta Neurobiol Exp (Wars) 2010;70(1):56-66.

10. Yeung J, Yaksh T, Rudy T. Concurrent mapping of brain sites for sensitivity to the direct application of morphine and focal electrical stimulation in the production of antinociception in the rat. Pain 1977;4(1):23-40.

11. Kieffer BL, Gavériaux-Ruff C. Exploring the opioid system by gene knockout. Prog Neurobio 2002:285-306.

12. Dualé C, Sierralta F, Dallel R. Analgesia induced by morphine microinjected into the nucleus raphemagnus: Effects on tonic pain. Curr Drug Deliv 2007;4(3):181-4.

13. Torrecilla M, Quillinan N, Williams J, Wickman K. Preand postsynaptic regulation of locus coeruleus neurons after chronic morphine treatment: A study of GIRK-knockout mice. Eur J Neurosci 2008;28(3):618-24.

14. Li Y, Liu X, Liu C, et al. Improvement of morphinemediated analgesia by inhibition of beta-arrestin 2 expression in mice periaqueductal gray matter. Int J Mol Sci 2009; 10(3):954-63.

15. Kuo T, Yang C, Chan J, Tsai H, Chan S. Further characterization of nociception-related and arterial pressure-related neuronal responses in the nucleus reticularis gigantocellularis of the rat. J Biomed Sci 1996;3(5):338-47.

16. Lipkowski A, Carr D. Rethinking opioid equivalence. PainClinical Updates 2002;10(4):1-7.

17. Bley K. Recent developments in transient receptor potential vanilloid receptor 1 agonist-based therapies. Expert Opin Investig Drugs 2004;13(11):1445-56.

18. Russell N, Schaible H, Schmidt R. Opiates inhibit the discharges of fine afferent units from inflamed knee joint of the cat. Neurosci Lett 1987;76(1):107-12.
19. Ferreira S, Nakamura M. II - Prostaglandin hyperalgesia: The peripheral analgesic activity of morphine, enkephalins and opioid antagonists. Prostaglandins 1979;18(2):191-200.

20. Coggeshall R, Zhou S, Carlton S. Opioid receptors on peripheral sensory axons. 1997;764(1-2):126-32.

21. Bergstrom J, Ahmed M, Li J J, Ahmad T, Kreicbergs A, Spetea M. Opioid peptides and receptors in joint tissues: Study in the rat. J Orthop Res 2006;24(6):1193-9.

22. Bigliardi-Qi M, Sumanovski L, Buchner S, Rufli T, Bigliardi P. Mu-opiate receptor and Beta-endorphin expression in nerve endings and keratinocytes in human skin. Dermatology 2004;209(3):183-9.

23. Zöllner C, Stein C. Opioids. Handb Exp Pharmacol 2007;177:31-63.

24. Tegeder I, Meier S, Burian M, Schmidt H, Gesslinger G, Lötsch J. Peripheral opioid analgesia in experimental human pain models. Brain 2003;126(5):1092-102.

25. Cunha T, Roman-Campos D, Lotufo C, Duarte HL, Souza GR, Verri WA, et al. Morphine peripheral analgesia depends on activation of the PI3Kgamma/AKT/nNOS/NO/ KATP signaling pathway. Proc Natl Acad Sci U S A 2010; 107(9):4442-7.

26. Nockemannl D, Rouault M, Labuz D, Hublitz P, MxKnelly $\mathrm{K}$, Reis FC, et al. The K+ channel GIRK2 is both necessary and sufficient for peripheral opioid-mediated analgesia. EMBO Mol Med 2013;5(8):1263-77.

27. Stein $\mathrm{C}$. The control of pain in peripheral tissues by opioids. N Engl J Med 1995;332(25):1685-90.

28. Stein C, Schafer M, Hassan A. Peripheral opioid receptors. Ann Med 1995;27(2):219-21.

29. Olsson Y. Microenvironment of the peripheral nervous system under normal and pathological conditions. Crit Rev 1990;5(3):265-311.

30. Zöllner C, Shaqura M, Bopaiah C, Mousa S, Stein C, Schafer M. Painful inflammation-induced increase in $\mu$-opioid receptor binding and G-protein coupling in primary afferent neurons. Mol Pharmacol 2003;64(2):202-10.

31. Jacobsen J. Topical opioids for pain \#185. J Palliat Med 2009;12(4):380-1.

32. Ramesh P. Topical morphine in Ayurveda. Palliat Med 1998;12(1):64.

33. Back I, Finlay I. Analgesic effects of topical opioids on painful skin ulcers. J Pain Symptom Manage 1995;10(7):493.

34. Zeppetella G, Joel S, Ribeiro M. Stability of morphine sulphate and diamorphine hydrochloride in Intrasite ${ }^{\circledR}$ gel. Palliat Med 2005;19(2):131-6.

35. Abdelmageed R, Labyad N, Watson D, Pournamdari M. Evaluation of the stability of morphine sulphate in combination with Instillagel. J Clin Pharm Ther 2008;33(3):263-71.

36. Huptas L, Rompoti N, Herbig S, Körber A, Klode J. A new topically applied morphine gel for the pain treatment in patients with chronic leg ulcers: First results of a clinical investigation. Hautarzt 2011;62(4):280-6.

37. Kumar R, Katare O. Lecithin organogels as a potential phospholipid-structured system for topical drug delivery: A review. AAPS PharmSciTech 2005;6(2):298-310.

38. D'Amour F, Smith D. A method for determining loss of pain sensation. J Pharmacol Exp Ther 1941;72:74-9.

39. González J. Modelos animales de dolor y aspectos éticos de la experimentación animal. Rev Soc Esp Dolor 2000;7:3138.

40. Kolesnikov Y, Jain S, Wilson R, Pasternak G. Peripheral morphine analgesia: Synergy with central sites and a target of morphine tolerance. J Pharmacol Exp Ther 1996;279(2):502-6. 
41. Kolesnikov Y, Chereshnev I, Pasternak G. Analgesic synergy between topical lidocaine and topical opioids. J Pharmacol Exp Ther 2000;295(2):546-51.

42. Ribeiro M, Simon J, Zeppetella G. The bioavailability of morphine applied topically to cutaneous ulcers. J Pain Symptom Manage 2004;27(5):434-9.

43. Watterson G, Howard R, Goldman A. Peripheral opioids in inflammatory pain. Arch Dis Child 2004;89(7):679-81.

44. Paice J, Von Roenn J, Hudgins J, Luong L, Krejcie T, Avram M. Morphine bioavailability from a topical gel formulation in volunteers. J Pain Symptom Manage 2008;35(3):314-20.

45. Gallagher R, Arndt D, Hunt K. Analgesic effects of topical methadone: A report of four cases. Clin J Pain 2005; 21(2):190-2.

46. Sylvester R, Schauer C, Thomas J, Steen P, Weisenberg A. Evaluation of methadone absorption after topical administration to hospice patients. J Pain Symptom Manage 2011;41(5):828-35.

47. Farley P. Should topical opioid analgesics be regarded as effective and safe when applied to chronic cutaneous lesions? J Pharm Pharmacol 2011;63(6):747-56.

48. Kirkham JJ, Dwan KM, Altman DG, Gamble C, Dodd S, Smyth R, et al. The impact of outcome reporting bias in randomised controlled trials on a cohort of systematic reviews. BMJ 2010:340-65.

49. Long T, Cathers T, Twillman R, O’Donnell T, Garrigues N, Jones T. Morphine-infused silver sulfadiazine (MISS) cream for burn analgesia: a pilot study. J Burn Care Rehabi 2001;22(2):118-23.

50. 50. Welling A. A randomised controlled trial to test the analgesic efficacy of topical morphine on minor superficial and partial thickness burns in accident and emergency departments. Emerg Med J 2007;24:408-12.

51. Skiveren J, Haedersdal M, Philipsen P, Wiegell S. Morphine gel $0.3 \%$ does not relieve pain during topical photodynamic therapy: A randomized, double-blind, placebo-controlled study. Acta Derm Venereol 2006;86(5):409-11.

52. LeBon B, Zeppetella G, Higginson I. Effectiveness of topical administration of opioids in palliative care: A systematic review. J Pain Symptom Manage 2009;37(5):913-7.

53. Flock P. Pilot study to determine the effectiveness of diamorphine gel to control pressure ulcer pain. J Pain Symptom Manage 2003;25(6):547-54.

54. Zeppetella G, Paul J, Ribeiro M. Analgesic efficacy of morphine applied topically to painful ulcers. J Pain Symptom Manage 2003;25(6):555-8.

55. Vernassiere C, Cornet C, Trechot P, Alla F, Truchetet F, Cuny JF, et al. Study to determine the efficacy of topical morphine on painful chronic skin ulcers. J Wound Care 2005;14(6):289-93.

56. Jansen M, Van der Horst J, Van der Valk P. Lack of analgesic effect from topical morphine in painful arterial leg ulcers. Proceedings from the Dutch Society of Clinical Pharmacy and Biopharmacy Meeting, April 13th, 2005. Br J Clin Pharmacol 2005;60:670-83.

57. Jansen M, van der Horst J, van der Valk P, Kuks P, Zylicz Z, van Sorge A. Pain-relieving properties of topically applied morphine on arterial leg ulcers: A pilot study. J Wound Care 2009;18(7):306-11.

58. Cerchietti L, Navigante A, Bonomi M, Zaderajko MA, Menéndez PR, Pogany CE, et al. Effect of topical morphine for mucositis-associated pain following concomitant chemoradiotherapy for head and neck carcinoma. Cancer 2002;95(10):2230-6.

59. Cerchietti L, Navigante A, Körte M, Cohen AM, Quiroga PN, Villaamil EC, et al. Potential utility of the peripheral analgesic properties of morphine in stomatitis related pain: A pilot study. Pain 2003;105:265-73.

60. Vayne-Bossert P, Escher M, de Vautibault C, Dulguerov P, Allal A, Desmeules J, et al. Effect of topical morphine (mouthwash) on oral pain due to chemotherapy- and/or radiotherapy-induced mucositis: A randomized doubleblinded study. J Palliat Med 2010;13(2):125-8.

61. Saroja G, Devi P, Namrata R. Oral morphine solution as an oral rinse or mouth gargle for mucositis pain. Indian J Palliat Care 2010;16(1):54-5.

62. Krajnik M, Zylicz Z. Topical morphine for cutaneous cancer pain. Palliat Med 1997;11(4):325.

63. Krajnik M, Zylicz Z, Finlay I, Luczak J, van Sorge A. Potential uses of topical opioids in palliative care-report of 6 cases. Pain 1999;80(1-2):121-5.

64. Twillman R, Long T, Cathers T, Mueller D. Treatment of painful skin ulcers with topical opioids. J Pain Symptom Manage 1999;17(4):288-92.

65. Flock P, Gibbs L, Sykes N. Diamorphine-metronidazole gel effective for treatment of painful infected leg ulcers. J Pain Symptom Manage 2000;20(6):396-7.

66. Ballas S. Treatment of painful sickle cell leg ulcers with topical opioids. Blood 2002;99(3):1096.

67. Abbas S. Diamorphine-Intrasite ${ }^{\circledR}$ dressings for painful pressure ulcers. J Pain Symptom Manage 2004;28(6):532-4.

68. Gallagher R. Methadone mouthwash for the management of oral ulcer pain. J Pain Sympt Manag 2004;27(5):390-1.

69. Platzer M, Likar R, Stein C, Beuble E, Sittl R. Topical application of morphine gel in inflammatory mucosal and cutaneous lesions. Schmerz 2005;19(4):296-301.

70. Porzio G, Aielli F, Verna L, Cannita K, Marchetti P, Ficorella C. Topical morphine in the treatment of painful ulcers. J Pain Symptom Manage 2005;25(6):304-5.

71. Wilken M, Ineck J, Rule A. Chronic arthritis pain management with topical morphine: Case series. J Pain Palliat Care Pharmacother 2005;19(4):39-44.

72. Ashfield T. The use of topical opioids to relieve pressure ulcer pain. Nurs Stand 2005;9(45):90-2.

73. Tran Q, Fancher T. Achieving Analgesia for painful ulcers using topically applied morphine gel. Support Oncol 2007;5:289-93. 